\title{
Análisis de la convivencia escolar en segundo ciclo básico de la comuna (municipio) de Tomé: percepción de los actores
}

\author{
Oscar Nail Kröyer* y Máximo Muñoz Reyes \\ Facultad de Educación, Universidad de Concepción
}

(Recibido 7 Julio 2011; Aceptado 15 Septiembre 2011)

\begin{abstract}
RESUMEN: El presente artículo muestra los resultados y conclusiones de un estudio sobre la convivencia escolar realizado en la comuna de Tomé, Chile. Esta investigación se realizó a través de la aplicación de cuestionarios en 10 escuelas municipales de enseñanza básica. Los sujetos de la muestra son los directivos de los establecimientos y los alumnos, profesores y familias de los cursos seleccionados. Los resultados muestran que los actores evalúan positivamente la convivencia escolar en los diferentes establecimientos, sin embargo el estudio refleja que existe una percepción más positiva por parte de los alumnos de los centros que de los otros agentes educativos. Por otro lado se debe destacar la mala percepción que existe del trabajo de los profesores en los establecimientos, siendo este fenómeno el principal problema para alumnos y apoderados. Además destaca la limitada participación de los apoderados en los espacios escolares. La importancia de este estudio radica en que entrega información para la mejora de la gestión de la convivencia tanto en las escuelas como en la comuna en su totalidad
\end{abstract}

Palabras clave: clima escolar, problemas de convivencia, participación.

Analysis of school life in the second basic cycle of the municipality of Tomé: perception of the actors

\begin{abstract}
This paper presents the results and conclusions of a study on school life made in the town of Tomé, Chile. This research was conducted through questionnaires applied in 10 municipal schools of basic education. The subjects of the sample are heads of the institutions and students, teachers and parents of selected courses. The results show that people assess positively to school life in different establishments, but the study shows that there is a more positive perception by students of the centers of other educators. On the other hand it should be noted that there is poor perception of teachers' work in the establishments and this phenomenon is the main problem for students and parents. It also highlights the limited parental involvement in school areas. The importance of this study relates is that provides information to improve the management of coexistence both in schools and the community as a whole.
\end{abstract}

Key words: school climate, problems of living, participation. 


\section{MARCO TEÓRICO}

\section{Convivencia escolar: conceptos y estudios}

El sentido de la convivencia se puede definir como el factor de cohesión de los grupos y las instituciones (Ortega, 2003). En opinión de la profesora Ortega, el término es entendido en una triple perspectiva. La primera se enmarca desde un punto de vista popular, la convivencia es comprendida como la forma en que una persona convive en un lugar físico, compartiendo diferentes escenarios, actividades, convenciones y normas para poder vivir sin conflictos. Los sujetos deben aceptar y respetar las ideas e intereses de los otros, favoreciendo la resolución de conflictos en forma dialogada. La segunda perspectiva hace referencia al contexto socio-jurídico, cuyo significado hace hincapié en la forma que la sociedad construye leyes laborales y cívicas, para que cada persona ejerza sus derechos como ciudadano y ciudadana sin discriminación o distinción de clase social, construyendo así una democracia justa. Desde una perspectiva psicológica y educativa hace referencia al análisis de sentimientos y emociones que deben existir para relacionarse con los demás, estos sentimientos están directamente relacionados con la empatía emocional y cognitiva. La cual se adquiere cuando la Educación y el desarrollo de la persona siguen pautas sociales de apego, reciprocidad afectiva y creación de criterios morales, reconociendo, a través de estas bases, al otro como semejante y diferente al mismo tiempo.

El clima es un concepto importado del ámbito de la geografía a las relaciones humanas. Es también una metáfora empleada en la Pedagogía. Se reconoce en la primera instancia como un concepto que trata de explicar el ambiente o atmosfera que se produce cuando las personas se relacionan entre sí. La idea de clima aparece unida a conceptos y planteamientos tan singulares y diversos como la Sociología y la Psicología ambiental, teoría ecológica, sistema social y de relaciones, procesos, cultura, instituciones, medio, etc. (Romero, 2005).

Por clima escolar se entiende el conjunto de interacciones y transacciones presentes en el proceso educativo en circunstancias determinadas, con rasgos tales como: un marco moral colectivo en una organización; determina actitudes, expectativas y conductas; es influenciado por la organización; y se presenta de distintas formas dependiendo de las prácticas y distintas unidades organizativas. Se refiere principalmente a los valores, actitudes y sentimientos reinantes que definen la atmósfera en la escuela (Poole, citado por Tuvilla, 2007).

El clima es un marco (setting) de referencia para las actividades de quienes participan en mayor o menor medida en él. Influye en las expectativas, actitudes y conductas de los mismos y a través de éstas, el clima afecta al resultado de las organizaciones (logro, satisfacción moral). El clima, a su vez, tiene su origen y es sostenido por las actividades que se llevan a cabo en la organización. Los factores estructurales y contextuales influyen sobre el clima de las organizaciones pero sus efectos son mediatizados por la práctica y los procesos organizacionales.

El concepto que integra tanto los factores individuales y contextuales que 
inciden en el aprendizaje y en el logro académico, es el de clima escolar, entendido como el conjunto de características psicosociales de un centro educativo, determinados por aquellos factores o elementos estructurales, personales y funcionales de la institución, que, integrados en un proceso dinámico específico, confieren un peculiar estilo a dicho centro, condicionante a la vez de los distintos procesos educativos (CERE, 1993. citado en : Alarcón \& Ramagnoli, 2006). El clima escolar refiere entonces a los altos niveles de desarrollo emocional y social de los alumnos y profesores, como a una mejor calidad de vida escolar. Aunque la escuela no es una isla y está entonces determinada por factores contextuales de índole económico-social, existen posibilidades o espacios de acción para las escuelas y los docentes, los cuales pueden intervenir en el contexto-escuela, es decir en su clima, a favor de condiciones de aprendizaje que promuevan el desarrollo social, afectivo y cognitivo de los estudiantes. En ese marco un rendimiento académico favorable, constituye un efecto secundario, pero necesario de esos cambios. (Alarcón \& Ramagnoli, 2006).

\section{Violencia escolar}

Uno de los factores que ponen en mayor riesgo la construcción de la convivencia es, sin duda, la violencia escolar. Se puede señalar que este fenómeno no es un problema nuevo y tal vez por ello parece ser algo socialmente aceptado. La violencia, en su sentido etimológico, indica fuerza, poder y ejercicio de la coerción. Podemos afirmar que la violencia ha existido en todas las sociedades, ya sea como medio de defensa o para dominar a otros individuos. La violencia ha penetrado en todos los rincones de la vida cotidiana, en las familias, las instituciones, los países y las relaciones sociales como por ejemplo, las laborales, las profesionales o las afectivas. Por lo que se podría considerar que la violencia escolar no sería más que un espejo de la sociedad en la que vivimos. Uno de los factores que incide en esta reproducción son los medios de comunicación que muestran escenas violentas en los dibujos animados, los noticieros, las novelas y las películas. Algunos individuos no pueden diferenciar lo que es realidad o ficción y parecen ser indiferentes e insensibles ante tales imágenes (Maldonado, 2004)

\section{Conflictos, violencia y agresividad en el contexto escolar}

Según la autora Rosario Ortega (2000), el conflicto surge cuando se juntan dos posiciones frente a una necesidad, situación, objeto o intención. Ahora bien, si en un conflicto los mecanismos que se utilizan para resolverlo no son los apropiados, puede surgir la agresividad y que, si esta no se controla, puede derivar en violencia. En este caso, la violencia se puede entender como un abuso de poder sobre la otra persona y que no tiene por finalidad resolver el conflicto sino más bien dañar al otro. A pesar de que el concepto de violencia está sometido a valores y costumbres sociales, siempre existe violencia cuando una persona impone 
su fuerza de manera injustificada y produciendo un daño en la víctima.

Un conflicto se da cuando dos personas entran en oposición o desacuerdo de intereses y/o posiciones incompatibles. Dependiendo de la forma en la que se resuelva este conflicto, la relación entre ambos puede terminar fortalecida o por el contrario dañada. Cuando se habla de resolución pacífica de conflictos se está haciendo referencia a la intención o voluntad de las partes en resolver una disputa sin recurrir a la violencia. Ambos sujetos considerarán los intereses del otro y estarán dispuestos a ceder en las posiciones personales para lograr una solución dialogada, en la ambas partes se beneficien. Es importante señalar que ambas personas poseen igualdad de derechos y oportunidades. El propósito de esta forma de resolver los conflictos es la de enriquecer la relación, por lo que esta posición, también implica reparación del daño, si ello fuera necesario.

En cambio, la agresión es una respuesta hostil frente a un conflicto. La agresión tiene relación con una conducta fuera de control, cuya finalidad es triunfar en la relación sin importar la integridad física o psicológica de la otra persona. La agresión se suele producir debido a la ausencia de una forma inadecuada de expresar los sentimientos, emociones, necesidades, percepciones, opiniones e intereses.

Por su parte la violencia se refiere a una situación límite en la que se hace uso de la fuerza produciendo daño en la otra persona.

\section{Estudios de convivencia escolar en Chile}

En el año 2009 el Ministerio de Educación de Chile (MINEDUC) realizó una investigación para conocer la percepción de los escolares respecto de la convivencia y violencia presentes en su entorno escolar, llamada III Encuesta Nacional de Violencia en el Ámbito Escolar. Dentro de sus resultados se observa que existe una buena o muy buena percepción de la convivencia escolar por parte de los docentes y alumnos. En el caso de los establecimientos municipales un $55,4 \%$ de los alumnos percibe que la convivencia es buena o muy buena, siendo el porcentaje más bajo en comparación con los alumnos de establecimientos particulares pagados y particulares subvencionados. Sobre la percepción de seguridad en el establecimiento escolar un $67,8 \%$ de los alumnos de establecimientos municipales afirma sentirse seguro. Este promedio vuelve a ser inferior a la percepción de los alumnos de establecimientos de dependencia diferente y además es inferior al promedio nacional que alcanza un $75 \%$. En términos nacionales un $84,7 \%$ de los docentes y $80,1 \%$ de los asistentes de la educación perciben que la relación entre docentes y alumnos es buena o muy buena. Por otro lado solo un $47 \%$ de los alumnos tiene esa percepción. Finalmente respecto de la agresión al interior de los establecimientos municipales un $25,8 \%$ de los alumnos ha sido agredido, siendo este porcentaje mayor al de los establecimientos de distinta dependencia y mayor al promedio nacional que alcanza un 23,3\%. (MINEDUC, 2009. III Encuesta Nacional de Violencia en el Ámbito Escolar). Por tanto, si bien la convivencia escolar es percibida como buena, los establecimientos muni- 
cipales tienden a tener una peor percepción de esta, lo que se vincula con los altos niveles de agresión presentes en este tipo de establecimientos.

La Fundación Paz Ciudadana realizó un estudio focalizado en la búsqueda de soluciones para la violencia escolar, llamado Violencia Escolar en Chile: Situación actual y Desafios (Fundación Paz Ciudadana, 2005). Este estudio realizó previamente una delimitación de los tipos de violencia y las consecuencias que esta provoca en las escuelas. Posteriormente el estudio identificó los tipos de agresión según la edad de los estudiantes, separado en dos grupos: el primero fue llamado victimización infantil, donde se encontraban niños de 7 a 10 años y que obtuvo como resultado que, por lo menos un tercio de los niños en este rango de edad, es víctima a lo menos una vez al año de agresiones psicológicas y/o físicas de parte de sus pares. Por su parte, el segundo grupo, denominado victimización juvenil, cuyo rango de edad era de 12 a 18 años, fueron las agresiones físicas las que se dan con más frecuencia por parte del grupo de pares. En este estudio se determinó además que el clima escolar en Chile es relativamente bueno y que los alumnos en su mayoría dicen sentir cariño por su establecimiento

Otro estudio es el llamado Primer Estudio Nacional de Convivencia Escolar, ejecutado por IDEA Chile (Instituto de Evaluación y Asesoramiento Educativo, 2005) en el año 2005. Solicitado por el Ministerio de Educación y por la UNESCO, fue aplicado en 507 establecimientos del país. Establecimientos educativos de Enseñanza General Básica y Media (científica, humanista y técnicoprofesional), ya sean municipales, particulares subvencionados o particulares pagados, con una muestra total de 41.729 alumnos y 6.782 profesores encuestados. Se pudo concluir que el $81 \%$ de los alumnos manifestó sentirse bien en el colegio y tener buenas relaciones personales con los demás. El porcentaje de alumnos que eran frecuentemente objeto de algún tipo de violencia por parte de sus pares, principalmente a través de insultos, fue de un $28 \%$. Por su parte un $9 \%$ de los estudiantes señalaron haber sido golpeados a menudo o siempre. La agresión física entre compañeros también presentaba diferencias, pues un $68 \%$ los alumnos que pertenecían al grupo socioeconómico bajo dijeron nunca ser golpeados, en cambio, un $76 \%$ de los alumnos pertenecientes al grupo socioeconómico alto dijeron nunca haber sido golpeados. Ahora bien, los alumnos presentaron una mayor tendencia a las agresiones y maltratos, que las mujeres. Por el contrario, el ignorar y el rechazar se daba más en las alumnas que en los alumnos. Se pudo comprobar que la agresividad y el maltrato se daban en mayor cantidad en la Enseñanza Básica que en la Media. Entre los estudiantes lo más frecuente era el insultar, el ignorar y el rechazar a otros, mientras que el robar o romper cosas a otros compañeros fueron los menos declarados. Por otro lado, los docentes percibían un mayor porcentaje de maltrato que los estudiantes (36\% frente al $70 \%$ que declararon nunca haber sido golpeados).

Del estudio anterior se puede destacar la alta valoración positiva de la convivencia escolar que realizaron los alumnos y profesores en relación al establecimiento, sus compañeros y las relaciones interpersonales que se dan en él. Del mismo modo un alto porcentaje del alumnado (85\%) y del profesorado $(88 \%)$ 
piensan que el establecimiento educacional es un lugar que enseña a relacionarse positivamente con el resto de las personas. Sin embargo, lo que sí presentó diferencias fue el sentirse bien en el establecimiento educativo y el tener muchos amigos pues los cursos de Enseñanza Básica señalaron tener una mayor satisfacción que los alumnos de Enseñanza Media.

Con respecto a la disciplina y la convivencia, un $74 \%$ del alumnado dijo que en sus establecimientos existe orden y $67 \%$ de ellos considera que las normas son adecuadas. El 21\% del alumnado, sobre todo los estudiantes de cursos superiores, señala que los profesores no tienen siempre el mismo criterio cuando aplican las normas, lo que es un factor que puede alterar la convivencia escolar. También el trato igualitario fue cuestionado pues 39\% del alumnado cree que todos los alumnos no son tratados de la misma manera. Lo más crítico entre los factores que alteran la convivencia escolar es la percepción de que el alumnado no tiene participación, es decir, que no se toman en cuenta sus opiniones a la hora de resolver problemas que se suscitan en el establecimiento. Así un 54\% de ellos consideraba que no tenía participación en la toma de decisiones. Este estudio, en términos generales, nos habla en términos de una buena convivencia escolar, pero debemos ser conscientes de la existencia de violencia escolar en los centros educativos chilenos, aunque su porcentaje sea bajo.

\section{METODOLOGÍA}

Este estudio se clasifica como no experimental, ya que se manipula al menos una variable independiente para observar su efecto y relación con una o más variables dependientes, siendo los grupos poblaciones escolares: alumnos, profesores, directivos y apoderados. La importancia de este estudio se fundamenta que para valorar la convivencia escolar participaron cuatro actores relevantes de las comunidades educativas, incluidas las familias, lo cual entrega una perspectiva más enriquecedora del estado de la convivencia escolar en los establecimientos participantes

\section{Objetivos de la investigación}

\section{Objetivo general}

Analizar el estado de la convivencia escolar en segundo ciclo básico de 10 establecimientos municipales de la comuna de Tomé a partir de la percepción de alumnos, apoderados, directivos y profesores

\section{Objetivos específicos}

1. Caracterizar la calidad de la convivencia escolar en los establecimientos escolares a partir de la percepción de alumnos, familias, directivos y profesores. 
2. Conocer las posibles diferencias en relación a la percepción de la calidad de la convivencia en función de los distintos actores educacionales.

3. Identificar los principales problemas de convivencia escolar que ocurren en los establecimientos escolares

4. Describir la participación de los padres en los distintos espacios que se generan al interior de los establecimientos escolares.

\section{Muestra}

El proceso de muestreo consta de dos etapas: en un primer momento se trabajó con una muestra intencionada que correspondió a 10 establecimientos básicos y municipales de la comuna de Tomé. Posteriormente se procedió a realizar un muestreo aleatorio estratificado de fijación porcentual de alumnos de segundo ciclo básico $\left(5^{\circ}\right.$ a $8^{\circ}$ básico $)$, un familiar de cada estudiante participante, profesores que realizan clases en ese nivel y el equipo directivo de los establecimientos, que corresponden a: director, inspector, jefe de Unidad Técnica Pedagógica y/u orientador). Se debe especificar que en los casos en que la población de alumnos de segundo ciclo básico de los establecimientos fuera menor a 50, se seleccionó al total del alumnado. La muestra total del estudio es de 1317 sujetos, lo que se detalla a continuación:

Tabla 1. Muestra de la Investigación

\begin{tabular}{|lccccc|}
\hline & Alumnos & Familias & Profesores & Directivos & Total \\
Establecimiento 1 & 89 & 89 & 16 & 2 & 196 \\
Establecimiento 2 & 19 & 19 & 8 & 2 & 48 \\
Establecimiento 3 & 122 & 122 & 19 & 5 & 268 \\
Establecimiento 4 & 31 & 31 & 9 & 2 & 73 \\
Establecimiento 5 & 22 & 22 & 6 & 2 & 52 \\
Establecimiento 6 & 31 & 31 & 6 & 2 & 70 \\
Establecimiento 7 & 25 & 25 & 4 & 1 & 55 \\
Establecimiento 8 & 43 & 43 & 12 & 1 & 99 \\
Establecimiento 9 & 132 & 132 & 17 & 5 & 286 \\
Establecimiento 10 & 76 & 76 & 14 & 4 & 170 \\
Total & 590 & 590 & 111 & 26 & 1317 \\
\hline
\end{tabular}

Instrumento de recolección de información

Cuestionario para valorar la Convivencia Escolar (Ortega \& Del Rey, 2003) 
en sus cuatro versiones: alumnado, familias, profesorado y directores en el que se explora, a través de preguntas cerradas, las distintas dimensiones de la convivencia escolar así como los problemas que la afectan. Entre ellos, la calidad de relaciones interpersonales, la comunicación, la disciplina e indisciplina, los conflictos y la violencia interpersonal.

A continuación se presentan las dimensiones del instrumento, con ejemplos de preguntas en cada una de ellas:

- Relaciones entre alumnos, entre maestros y alumnos con maestros: ¿Cómo te llevas con tus compañeros?, ¿Qué percepción cree que tienen de usted sus colegas?

- Participación de los apoderados en las actividades del colegio: ¿En cuál de las siguientes participan las familias?

- Percepción que tienen sobre la aceptación de la que disfrutan los estudiantes, por parte de sus pares y de sus maestros, y los maestros por parte de sus pares y estudiantes: ¿Qué opinión piensas que tienen de ti los profesores?, ¿Qué opinión cree que tienen de usted sus alumnos?

- Violencia: ¿Los Alumnos se pelean? ¿Los Alumnos se insultan?

- Normas: ¿Existen diferencia en las normas de clase entre uno y otro profesor?

\section{Análisis de confiabilidad del instrumento}

Para verificar la confiabilidad de los instrumentos respecto de sus dimensiones, se utiliza el estadístico Alfa de Cronbach (basado en Coeficiente de Correlación de Spearman debido a la característica ordinal de los datos), para determinar si existe consistencia interna. La razón por la cual se utilizó Coeficiente de Correlación de Spearman, que es una herramienta estadística no-paramétrica, es que, como las respuestas están dadas en escalas ordinales, las observaciones no se pueden asumir como continuas y mucho menos normales.

El valor del estadístico Alfa de Cronbach para el grupo total de encuestados (alumnos, apoderados, profesores, directores) y considerando las dimensiones de interés asume el valor $\alpha=0,71$. Se concluye, por tanto, que existe consistencia interna en el instrumento.

\section{Análisis de la información}

Se realizó un análisis descriptivo de las dimensiones, dirigido a analizar la calidad de la convivencia escolar en los establecimientos escolares a partir de la percepción de alumnos, apoderados, directivos y profesores y a analizar el clima 
laboral en los establecimientos escolares a partir de la percepción de directivos y profesores. Para esto se utilizaron medidas, es decir, construcción de índices, que corresponden a promedios de los valores codificados de las respuestas por ítems (dimensiones).

Aunque las observaciones no corresponden a una escala continua, los promedios aportan una buena descripción de las opiniones, en función de cuan cerca o lejos están de los valores enteros correspondientes a cada concepto (1 Bueno, 2 Regular, 3 Normal, 4 Malo)

Además se construyeron gráficos circulares y gráficos de líneas, para comparar valores promedio de respuestas por grupos y porcentajes de prevalencia de ciertas características, y así ilustrar las conclusiones.

\section{RESULTADOS}

Análisis global de categorías

Tabla 2. Promedios Totales por Actor y Categoría

\begin{tabular}{|ccccc|}
\hline & Relaciones & Participación & Normas & Global \\
\hline Alumnos & 1.69 & 1.54 & 1.8 & 1.68 \\
Familia & 1.49 & 2.03 & 1.78 & 1.77 \\
Profesores & 1.51 & 2.12 & 2.28 & 1.97 \\
Directivos & 1.39 & 2 & 2.16 & 1.85 \\
Global & 1.52 & 1.92 & 2.01 & \\
\hline
\end{tabular}

Nota: 1: Bueno; 2: Normal; 3: Regular; 4: Malo

Como se puede observar en el cuadro 1, tanto alumnos, familia, profesores $\mathrm{y}$ directivos tienen en promedio una percepción Buena de la convivencia en sus establecimientos, sin desmerecer que en los casos de profesores y directivos, la evaluación de las categorías Participación y Normas supera los límites del rango Bueno con promedios que alcanzan 2.12 y 2.28 para los profesores; y para los directores los valores alcanzan 2 y 2,16 en las categorías antes mencionadas. En esta misma línea, las familias tienen un promedio superior al rango Bueno en la categoría Participación, con una media que alcanza 2,03. Por otro lado la mejor percepción la tienen los alumnos con un 1,68 promedio, seguidos por las familias con un 1,77 , los directivos con un 1,85 y finalmente los profesores con un 1,97. Este último puntaje marca el límite superior de los actores y se sitúa solo 0,03 
puntos bajo Normal. Es importante destacar la percepción de los docentes, quienes a pesar de considerar Buena la convivencia, tienen una diferencia importante en su puntaje promedio con el resto de los actores y a su vez, estos últimos tienen diferencias de puntaje entre sí.

En cuanto al análisis por categorías, la primera de estas (Relaciones) es la que tiene el puntaje más bajo con un 1,52. De esta manera se puede concluir que los actores no observan que las relaciones interpersonales sean un problema grave en sus establecimientos. Por otro lado la categoría Participación tiene un puntaje de 1,92 estableciéndose solo 0,8 puntos por debajo de Normal. En esta dimensión los profesores y las familias son los que tienen la peor evaluación con 2,03 y 2,12 respectivamente seguido de los directores con 2 . Todos estos actores califican, en promedio, la Participación como Normal y solo los alumnos la califican como Buena, a pesar de esto la media global de la categoría sigue encontrándose dentro del rango de la mejor evaluación. Esto se debe a que los promedios aritméticos se ven altamente afectados por sus valores límites.

Finalmente la categoría normas tiene un puntaje de 2,01 encontrándose dentro del rango de Normal. Esta es la categoría con la peor percepción por parte de los actores en particular los profesores y directivos quienes tienen una evaluación promedio de 2,28 y 2,16 respectivamente; le siguen alumnos con un 1,8 y familias con 1,78. Nótese que en esta categoría todos los puntajes se encuentran más cercanos al rango Normal y nuevamente son los profesores los que evalúan de manera más crítica la categoría. De manera global, los investigadores apreciaron diferencias significativas en las respuestas de los distintos actores según establecimiento.

El siguiente figura muestra de manera visual lo anteriormente expuesto:

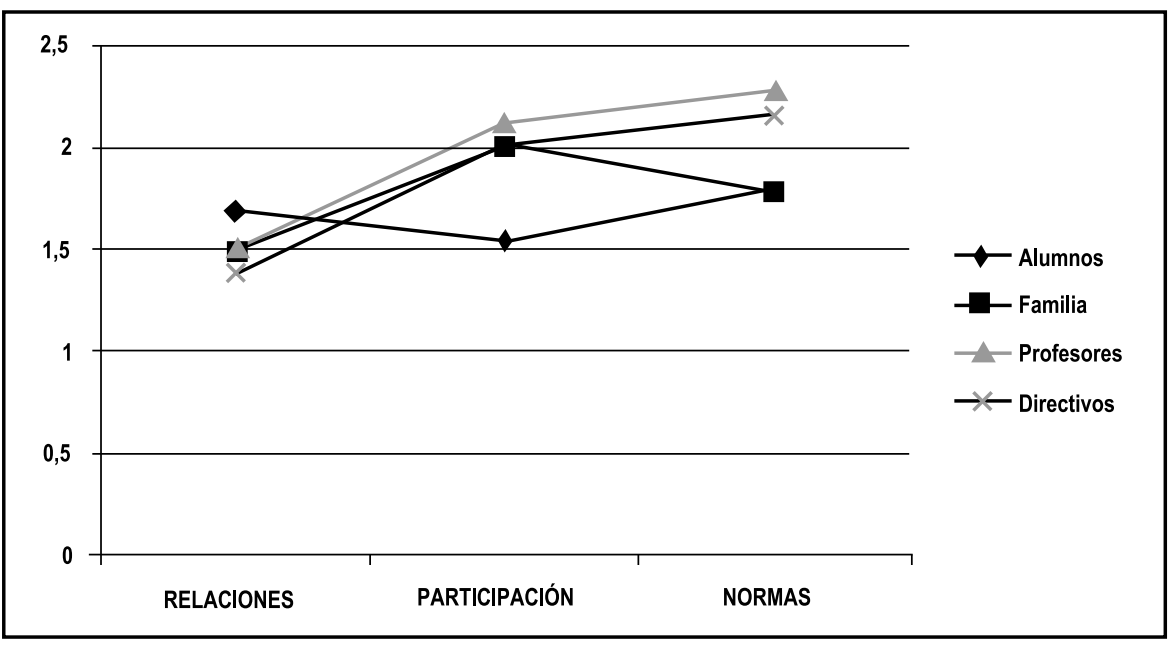

Figura 1. Comparación entre actores y categorías. 
Se observa en la figura que en los alumnos evalúan de buena manera la Participación. Por su parte las familias y los alumnos evalúan de buena manera las Normas. Tanto profesores como directores muestran una clara tendencia a evaluar de manera negativa la Participación y las Normas. Además se puede observar las fluctuaciones de puntaje existentes en alumnos y familia, quienes evalúan de mejor o peor manera la Participación.

\section{Principales problemas de convivencia}

A continuación se presentan los problemas de convivencia identificados, su ocurrencia y el impacto que los actores dan a estos.

Tabla 3. Frecuencia de Aparición de Problemas de Convivencia por Actor

\begin{tabular}{|c|c|c|c|c|c|c|c|c|}
\hline \multirow[b]{2}{*}{ FENÓMENO } & \multicolumn{2}{|c|}{ Alumnos } & \multicolumn{2}{|c|}{ Familias } & \multicolumn{2}{|c|}{ Docentes } & \multicolumn{2}{|c|}{ Directivos } \\
\hline & $x$ & $\sigma$ & $x$ & $\boldsymbol{\sigma}$ & $x$ & $\boldsymbol{\sigma}$ & $x$ & $\boldsymbol{\sigma}$ \\
\hline $\begin{array}{l}\text { Enfrentamientos } \\
\text { alumno-profesor }\end{array}$ & 2,03 & ,846 & 2,01 & ,886 & 1,93 & 618 & 2,44 & ,177 \\
\hline $\begin{array}{l}\text { Malas palabras } \\
\text { en clases }\end{array}$ & 2,28 &, 869 & 2,19 & 1,014 & 2,47 & 682 & 2,19 & ,144 \\
\hline $\begin{array}{l}\text { No se respetan } \\
\text { las normas }\end{array}$ & 2,72 & ,802 & 2,51 & ,933 & 2,82 & ,557 & 2,56 &, 540 \\
\hline $\begin{array}{l}\text { Los alumnos } \\
\text { se insultan }\end{array}$ & 2,7 & ,864 & 2,65 & ,934 & 2,83 & ,650 & 2,63 & ,479 \\
\hline $\begin{array}{l}\text { Los alumnos } \\
\text { se pelean }\end{array}$ & 2,55 & ,824 & 2,54 & ,879 & 2,76 & 634 & 2,31 & ,479 \\
\hline $\begin{array}{l}\text { Hay grupos que } \\
\text { no se llevan bien }\end{array}$ & 2,82 & ,873 & 2,80 & 955 & 2,68 & 660 & 2,31 & ,479 \\
\hline $\begin{array}{l}\text { Hay niños que no } \\
\text { se integran y se } \\
\text { sienten solos }\end{array}$ & 2,23 & 1,023 & 2,42 & ,961 & 2,39 & 639 & 1,94 & ,479 \\
\hline $\begin{array}{l}\text { Los profesores hacen } \\
\text { lo estrictamente } \\
\text { necesario }\end{array}$ & 3,18 &, 813 & 3,05 & ,927 & $, 2,49$ & 844 & 2,56 & ,498 \\
\hline $\begin{array}{l}\text { Los alumnos piensan } \\
\text { que los profesores no } \\
\text { los entienden }\end{array}$ & 2,79 & ,928 & 2,84 & ,821 & 2,89 & ,783 & 2,38 & ,668 \\
\hline $\begin{array}{l}\text { Los alumnos están } \\
\text { desmotivados, se } \\
\text { aburren }\end{array}$ & 2,59 &, 867 & 2,59 & ,875 & 2,54 & ,655 & 2,69 & ,479 \\
\hline
\end{tabular}

Nota: 1: Nunca; 2: Escaso; 3: Regular; 4: Mucho

La tabla 3 presenta presentan las medias aritméticas (x) y las desviaciones estándar $(\boldsymbol{\sigma})$ por actor, para una serie de problemas de convivencia escolar. En 
su mayoría de las medias aritméticas se encuentran entre los valores 2 y 2,99, lo cual permite observar que en promedio las dificultades presentadas ocurren escasamente en los establecimientos. Las excepciones las presentan los Docentes en el problema $n^{\circ}$ 1: "Enfrentamientos Alumno-Profesor", el cual se encuentra dentro del rango 1, lo cual indica que en promedio para los profesores, la aparición de este problema es casi nula. Lo mismo ocurre para los Directores en el problema $\mathrm{n}^{\circ} 7$, los cuales manifiestan que existen escasos problemas de integración social de los alumnos.

Por otro lado para los Alumnos y las Familias el problema $n^{\circ} 8$ "Los profesores hacen lo estrictamente necesario" se presenta de manera frecuente, lo cual llama la atención ya que son los estudiantes y sus familias los principales beneficiarios de la labor docente. Esta apreciación del trabajo de los profesores debe ser destacada, ya que implica algún tipo de disociación entre lo que los docentes realizan y las expectativas y valoración que los padres y los estudiantes tienen de su labor.

Para concluir, se destaca que todos los promedios totales se encuentran dentro del rango de lo escaso, lo cual si bien no significa que no existan problemas y/o que estos no merezcan estrategias de prevención y solución, se reconoce en los establecimientos un nivel de conflictividad y de problemas bajo entre sus actores.

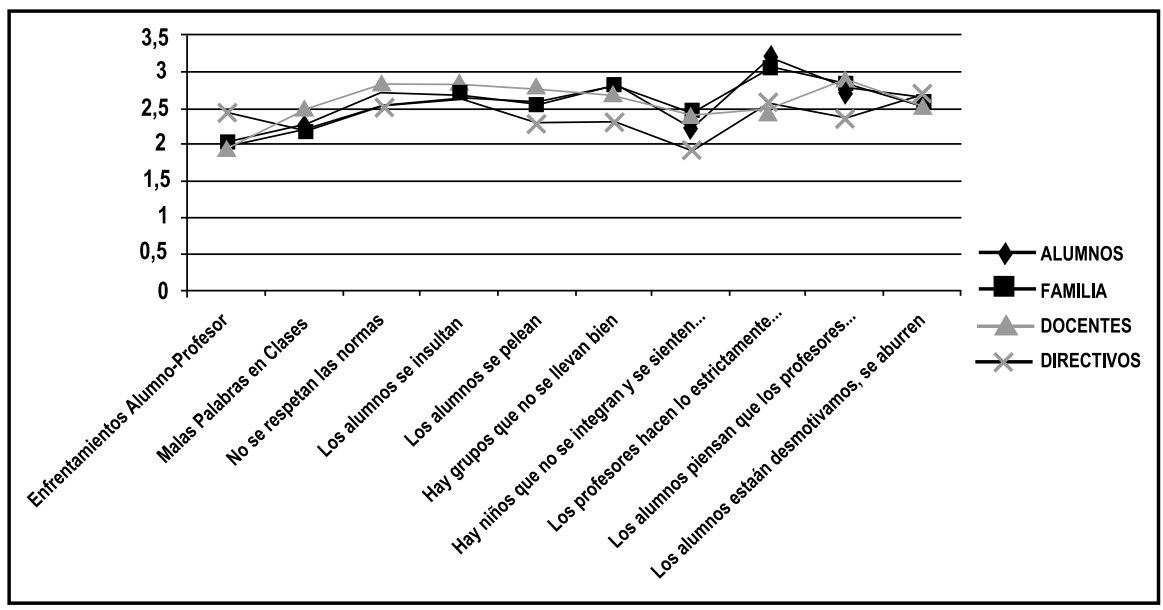

Figura 2. Comparación por actor y problemas.

La figura 2 permite comparar los promedios por problemas y actores

Se observa una tendencia común entre los actores al momento de evaluar los problemas. La gráfica de los Directivos en el problema $\mathrm{n}^{\circ}$ 7: "Hay niños que no se integran y se sienten solos" rompe esa tendencia, al igual que los Alumnos y Familias en el problema n ${ }^{\circ}$ : "Los profesores hacen lo estrictamente necesario". Sin embargo, la gráfica muestra una clara tendencia por parte de los actores a reconocer una baja aparición de los problemas presentados. 


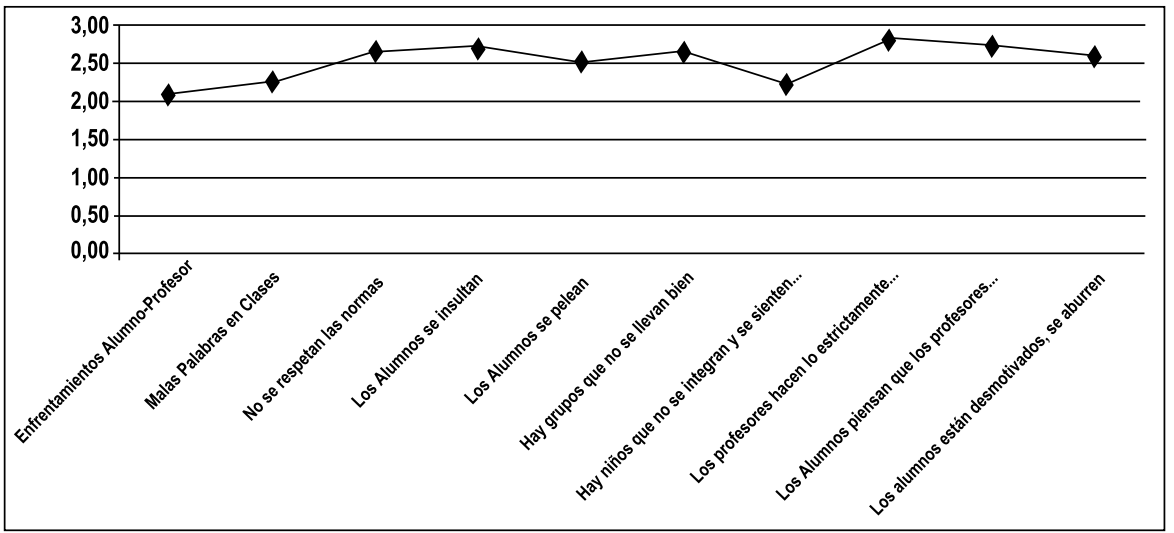

Figura 3. Frecuencia de aparición global de los problemas.

La figura 3 muestra los promedios globales de respuesta de los distintos grupos en relación a su percepción sobre la frecuencia de aparición de los problemas. Es posible observar que todos los problemas se encuentran entre los valores 2 y 3 , por lo cual su frecuencia de aparición es escasa. El problema de más alto puntaje, y por tanto de mayor aparición, es aquel que dice relación con que los "Profesores hacen los estrictamente necesario" y el de menor puntaje y frecuencia es aquel vinculado a los "Enfrentamientos alumno-profesor".

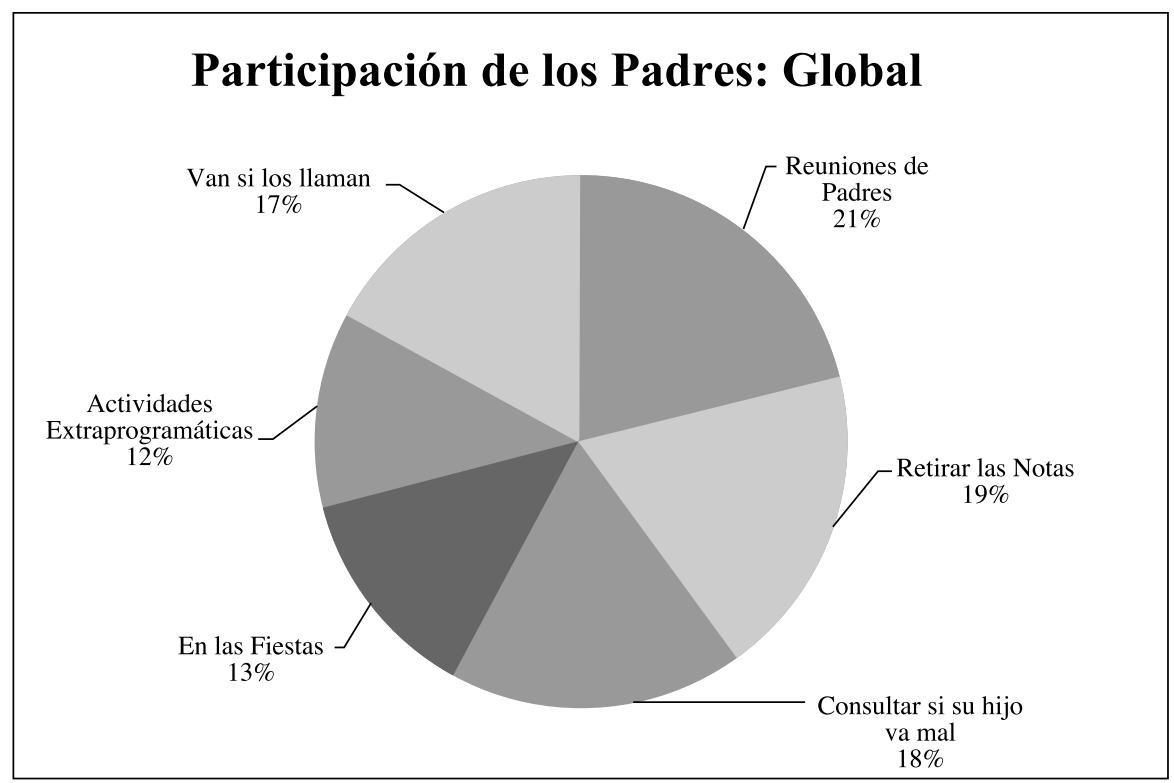

Figura 4. Participación de los padres global. 
La participación de las familias al ser considerada de manera global (promedio de la participación según los cuatro actores encuestados) deja en claro que para los actores la mayor participación de las familias ocurre en actividades obligatorias. La asistencia a reuniones, a retirar las notas, a consultar sobre el desempeño y comportamiento de sus hijos; y acudir si son citados ocupan los primeros lugares, y en algunos casos, tienen diferencias significativas con las actividades de participación libres e informales.

\section{CONCLUSIONES}

Existe de manera transversal una buena percepción general de la convivencia escolar al interior de los establecimientos por parte de todos los actores. Sin embargo se debe destacar que tanto docentes como directivos tienen una mirada más crítica en las categorías de Participación y Normas. Por otro lado los directores en términos generales tienen una percepción de la convivencia y de los problemas relacionados con esta disociada del resto de los actores participantes, la cual tiende en el caso de los índices generales a ser peor y en el caso de los problemas de convivencia a ser mejor

El principal problema de convivencia está relacionado con la percepción que alumnos y apoderados tienen del trabajo docente. Esto es significativo, ya que plantea un escenario de posible falta de compromiso o de habilidades en los profesores con su trabajo y con los principales beneficiarios de este último. Por otro lado los enfrentamientos alumno-profesor no son un problema significativo al interior de las escuelas, así como tampoco la violencia entre alumnos. Si bien estos problemas ocurren y tienen impacto negativo en la convivencia escolar, su frecuencia de aparición es baja.

Fomentar la participación de los padres y las familias en actividades de esparcimiento, de relaciones informales y no directamente relacionadas con el aprendizaje de los niños, se revela como fundamental para los establecimientos de la comuna. La inclusión y participación no solo se debe fundamentar en sentirse obligados a acudir o realizar tal o cual acción, sino que además debe tener como base la participación libre y voluntaria, para así construir identidad entre los apoderados y las escuelas de sus hijos

Además, se hace necesario trabajar la normativa de convivencia de una manera más democrática e inclusiva, para fomentar la construcción de valores y actitudes positivas para el desarrollo de los estudiantes, Por tanto no deja de ser necesario perfeccionar de manera constante la normativa de convivencia en los establecimientos y establecer procesos claros para su construcción.

Se considera necesario capacitar al profesorado en aspectos que dice relación con el mejoramiento de las relaciones profesor alumno al interior del aula, especialmente respecto a su manejo y desempeño de las clases desde el punto de vista de sus competencias que dicen relación con la gestión de la convivencia en el aula. En este sentido, otra comuna de Concepción, estamos aplicando experimentalmente una línea de intervención llamada "análisis de incidentes 
críticos de aula" .Los primeros resultados nos indican que es una buena herramienta de intervención, ya que apunta a desarrollar competencias más analíticas y reflexivas en los docentes respecto a los incidentes que le interfieren sus clases regularmente. A través de esta técnica los docentes aprenden a enfrentar futuras problemáticas de aula con mecanismos más pedagógicos y mejora su "autoridad" docente dentro del aula, como también la comunicación con el alumnado, aspecto fundamental en el mejoramiento de la gestión de la convivencia de aula.

\section{REFERENCIAS}

Alarcón, C. y Ramagnoli, C. (2006). El Clima Escolar como Plataforma de Cambio. Documentos UC. Santiago: Pontificia Universidad Católica de Chile.

Fundación Paz Ciudadana (2005). Violencia escolar en Chile: Situación Actual y Desafios. http://ramonburgos.tripod.com/sitebuildercontent/sitebuilderfiles/ ViolenciaEscolar.pdf

IDEA Chile (Instituto de Evaluación y asesoramiento Educativo) y UNESCO (2005). Primer Informe Nacional de Convivencia Escolar. La opinión de estudiantes y docentes. Santiago: MINEDUC.

Maldonado, H. (2004). Convivencia Escolar, Ensayos y Experiencias. Editorial Lugar. Buenos Aires.

MINEDUC. (2009). III Encuesta Nacional de Violencia en el Ámbito Escolar. Santiago: Chile.

Ortega, R. (2000). Educar la convivencia para prevenir la violencia. Madrid: Antonio Machado Libros S.A.

Ortega, R. (2003). La Convivencia en las Aulas: Problemas y Soluciones. Córdoba: Instituto Superior de Formación del Profesorado.

Ortega, R. y Del Rey, R. (2003). Cuestionario para Valorar la Convivencia Escolar. Universidad de Cordoba:Autor.

Romero, G. (2005). Hacia una pedagogía de contexto. Proyecto Filosofía para Niños y Niñas en el clima social. Universidad de Alcalá. España.

Tuvilla, J. (2007). Convivencia escolar y resolución pacifica de conflictos. Consejería de Educación y Ciencia. Junta de Andalucía. 